tation de chaleurse poursuit très loin, sans variations sensibles, d'où l'on peut conclure que, à une profondeur de 60 kilomètres, notre globe doit être à une temperature de $2000^{\circ}$, c'est-à-dire suffisante pour amener à l'état de fusion les substances les plus réfractaires. Mais qu'est-ce que cette prolondeur de 60 kilomètres. relativement aux dimensions d'une sphère de 6366 kilomètres de rayon? Nous voynns qu'elle en représente à peine la centième partie.

L'astronomic elle-même, et ce qu'on pourrait appeler la chimie sidérale, nous fournissent aussi des arguments de premier ordre. En effet, le spectroscope a permis de connaitre la nature des substances qui composent l'enveloppe incandescente du soleil. Or, parmi les nombreux corps simples révélés par les raies du spectre solaire, il ne s'en trouve qu'un seul qui ne soit pas encore connu sur terre. Ainsi donc, les deux astres, malgré leur disproportion relative, sont composés des mêmes éléments, ce qui rend à peu près évident une origine communz. De plus, le même moyen d'information a pu être appliqué aux étoiles, et a permis de les ranger en catégories. Ainsi tous les centres stellaires, dont la parenté avec le soleil est depuis si longtemps reconnue, sont le siège d'une même évolution, caractérisée par une diminution continuelle de l'énergie lumineuse, calorifique ou chimique.

Enfin, une dernière démonstration de l'existence de la chaleur centrale nous est fournie par les observations des géologues, établissant que, au moins jusqu'à la fin des temps primaires, la surface du globe jouissait d'un climat uniformément tropical. La flore houillère, qu'on la' recueille près des pôles, ou dans la zone équatoriale, apporte à cet égard des arguments décisifs, et les polypiers constructeurs tiennent cxactement le même langage. Les plantes décotylédones, dont l'organisation accuse le jeu des saisons, n'ont apparu quau milieu de l'ère secondaire, et ce n'est que vers la fin des temps tertiaires que les climats, dans nos contrées, ont commencé à se différencier.

pour formuler des explications, certains invoquent un aure mode de répartition des terres et des eaux, oubliant que, si les causes géographiques peuvent modifier quelque peu la distribution dẻs climats, elles sont impuissantes à supprimer le froid des régions polaires. D'autres font intervenir la chaleur centrale, dont le rayonnement ê̂t été autrefois plus actif. Une seule explication convient à tout; c'est celle qu'à donnée, il y a une vingtaine d'années, le $\mathrm{D}^{r}$ Blandet. Cette hypothèse consiste à admettre que le soleil ne formait, durant les temps primaires, qu'une nébuleuse très dilatée, enveloppant et baignant en quelque sorte la terre dans un faisceau de rayons, qui versait sur elle une chaleur et une lumière beaucoup plus douces qu'aujourd'ui, mais convergentes et parcourant une plus faible distance. De la sorte, aucun point de la terre n'était condamné à ces longues nuits qui pèsent aujourd'hui sur les hautes latitudes. Il n'y avait ni zônes de climats, ni saisons tranchées, et ces différences ne se sont produites que lorsque, à force de de se contracter par la condensation, le soleil a diminué de diamètre, pour n'apparaître plus que comme un petit disque brillant. Or, d'une part, cette manière de voir est unc conséquence directe de l'hypothèse de Laplace, d'autre part elle est en rapport avec la théorie d'Helmholiz, qui admet que la condensation progressive de l'astre central peut seule assurer la conservation de son cnergic calorifique.

Ainsi, nous possédons maintenant des preuves qui nous convainquent de l'existence du feu central. Ces six preuves: le mode de formation de la terre; l'étude de sa forme en relation étroite avec les phénomènes qui lui ont donné naissance; sa densité; le résultat des expériences concluantes relativement à l'augmentation de la chaleur avec la profondeur; les données spectroscopiques; enfin l'uniformité de la tore des temps primaires, nous permettent, étant donné qu'elles s'accordent parfaitement avec la nature chimique de la croûte terrestre, et avec l'état minéralogique des terrains primitifs, d'arriver logiquement aux causes des phénomènes volcaniques.

(A suivre.)

Jean Escard.

\section{CONGRÉS INTERNATIONAL DE MARSEILLE des Applications de l'Électricité}

\author{
$(S w 2 / c)$
}

CINQUIEMIE SECTTON

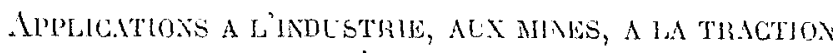
ET A L AGRICULTURE

Présidenl : M. Bolicherot.

Tice-Présidents : Docteur Trs5or, M. LEGouez. Secrélaire: M. Routr.

Séance du mardi lj seplembre.- M. Buuxsmari donne lecture de son raploot sur L'équipement électrique des mumes et maluque l'espril dans lequel al a congu son rapporl.

Lassant de còté l'étude dólallée des slalions contrales, saul malloalion des grandes lighes vers lesquelles celle étude somente (usage des turbnnes et des moleurs à gaz), lo raj)porlen ébablit une classincalion des duers gemres d'aphareils :

Instaliations du pour. - Pompes. - Ventulaleurs. - Moteurs au fond. - Traction uu fond. - Iiclairage au fond. -.. Erlraction. - Au fur el ì mesure de lexamen de chacun do ces points, M. Brtwswar signale somlement les pounts particuliers sur lesquels lattention du constructeur ou de liexpioilant doul se porter.

Passant rapidement sur les six premières catégories, ll entre dans l'analyse sommanre du rapporl, dont le volumo ne permel pas la leclure, et marloulièrement la question st importante de lextracuón électrique. L'examen des divers systèmes en présence porte sur le principe des divers moyen: de commandes, sur la comparason des rendements d'exploslation et du coút de premer établissement.

En cours, lo rapport élablit les avantages comparalifs du couranl continu et du courant polyphasé.

M. Leprixce-Ringuet, fait observer que Iapplicalion du principe de Davy, aux nuoteurs des apparells pilacés dans les milleux grisouleux, esl me jnvention d'orlgune française qu a été signalée à léproque dans les publucations techniques et rue l'achmnstralion supérieure ne s'est jamais désintéressée des rpplicallons générales de l'électricile dans les mines, témon les expérionces en cours ou projelées dans

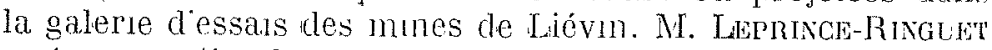
mot en question la perfechou des apliludes des moteurs ćleotriques tripliasés applifućs diroctement à l'oxlraction.

M. Dunousin communique un ecrain nombre d'observa. tions fort interessantes sur Jes pounls suivants

$1^{\circ}$ Lemploi des Lurbmes à vapeur déchapdroment dans les sièges d'extraction ;

$2^{\circ}$ Supéroribé des couronls liphasés, el adaulation parliculièromenl avanlageuse de la Jasse fréfuence (fréquence 25), sumloul pour les commandes directes d'amparels d̀ faible vitrese de rotation:

$3^{\circ}$ Imporlance scomalaire au poinl de vuc consommalion de vapeur dans la sulystilulion de l'ćlectricile à la vapour dans l'extraction, vu lópuivalence pratrpue des deux syslèmes, mais intérel prínondérant de lédectricilé au pount do vue de la condule el do ladaphalion des apparens do sćcurité.

M. Lubert soulient les avantages du courant continu of raison de la souplesse quion présenle l'apmicalan of onnleste, en sappuyant sur des résullats dexploslatson frobos-

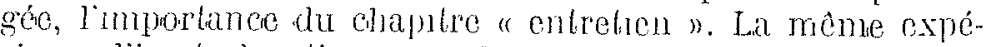
rience lincule a esumor que les sujétıons dos aponrcils tripllasés el leur enlnelion conduisent à une exploilaluon phus oncrouse.

M. Aubent pose également la ruestion de l'adaptalion des moteurs trıphasés à la commande de cerlains appareils spé- 
mand an mines, lols que los compmesseurs, par oxemple. Quanl a l'extracilion, M. Aubent indique que le chorx du imule dexbation doul ctre envisagé au triple point de vue the la dépense de vapour, de la docilıté el de la sécurıté.

V. Do molsm, après une rópliquc du rapporteur, appue sor allirmations on faveur des courants triphasés el signalc une solubion, bascé sur ture modiflcation du diagramme 11 Hhmssion, nermetlant la marche a vitosse constante des comprosicurs et, par conséquent, la possibulité demplon des moleurs triphasés.

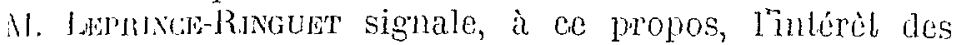
fuho-comllresseurs à commande dectrique.

1. JNAN donne lechure de son rapport sur l'Applicalion de lit briblion aux chemins de fer.

Sisance du mereredi 16 septembre. - M. Satvage présente son rapport sur l'Appluation des umlés multiples it la traclion électrique. Il explique comment les unites mulhules jurísenteul des avantages considérables sur les locomoluves ou les unités doubles pour les appilucalions de la trachom forolricfue sur les lignes métropolitanes ef de banlieue. Il msisle sur la supériorilé des systèmes à automatrcité indvaluelle, dont l'emploi se généralise de plus en plus. M. Sat$\checkmark$ vir lermune par lapplicatron des unités multiples à la lradion раг courant alternalif simple, en concluant par me prórience pour le réglage de la tension par régulateur d'unthethen.

il. JANin indrque quion peut avee l'exploilation électrique Iransporter 20.000 personnes à l'heure.

M. T'rssot indicque qu'il y a intérêt pour la traction sur grmiles lignes à employer un tracteur unique.

V. DF TRAz résume son rapport sur lEtude du fonctionnement des moteurs de traction à rourant continu. Le principe des mithodes gu'll expose consiste à établir, une fois pour lonles, la courbe des variations, en fonction de la vilesse du tran, de lintensilé quil demande eb de I'accélération qu'll quil prend on palıer. Celle dernière courbe donnera les alocklúrallons hors des paliers par simple déplacement Héhelle. Ayant cetle base, il est facile déscrire comjhetement la marche din train dans un intervalle de stathons fommé, amsi que sa consonnation, ef M. DF TRAz signale plusteurs applicalions de ces méthodes, nolamment pour lia dólomination de la conduite économiçue ou pour Jes jorojels de traction.

M. Mazs dil quelques mols sur la question de l'adhérence a des accélératrons qui, pour des raisons économiques, 110 jouvont dépasser certains clillires.

M. Sklictomicz fat observer que cesl la dérivce de l'accé beration qui a, pour les voyageurs, une influence sur le confort.

M. wa Thw retmond ru il est de lavis de M. Selutowicz et ynil n'y a pas lou do limiter les accédéralions mais d'apporter des perfocliomnements, par exempie par lo démarrayo automblique, à la bonne succession des variations d'efforls au clébut de la marche.

M. Rot row résume son rapport sur Les régulateurs automatuques el les semo-moteurs blectriques.

Il examine, en particulıe, le cas d'un groupe électrogène mwin d'un rógulateur automalique de vitesse, et d'un régulafrur de tension indépendants, il montre que la stabilité de l'ŕfulibre qui dol rxister à l'étal de régime entre lo couple moleur ot lo couple résistanl peut alors ilre compromise. baus foutes les apphlicalions du réglage indrect, lorgane

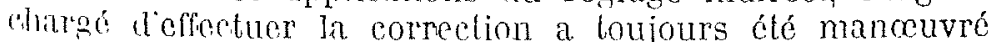
aver. tmo vilesse constante : par contre, lorsque le réglage at effechué à la main, Jlomme, régulaleur intelligent, agit loujours aver uno vitesse proportiomolle à l'écort gu'il s'agit in corriger.

Il est possihle rle róaliser des régulaleurs automatiques dommat une vitesse de manceuvre varjalule. Nr. Routw indifur Ios résultals auxquets on est conduil par le calcul dans les deux cas parlianins ci-apres : vilesse de manomure pro-

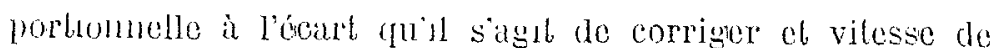
mancouvre proportionnelle à l'accélération alu groupe. Al. Routen termune en signalant lintéròt du réglage cent tral gue l'omphoi d'un servo-moleur éloctruque permet de gúnéraliser au cas de pusieurs usmes alornentant un mèmo l'óseau.

M. MARCer BnuLt préscnle un disposilf de démaringo anomalique jour les moleurs électriques de lous gonles. It en expose le principe basé sur l'uthlisation de conduchours de seconde classe paces aux bornes de résistances tle démarrage ; ces éléments en sechatiant motlent automall. ruement on court-circuit la résistance de démarrage. Il indicue l'application particulière de ces résistances aux moleurs dinduction dans lescuels al permet la summession dos bagues et organes de démarrago. Il termme par la descrij. tion d'un appareil de déniarrago basé sur ce principe et lindication de ses condulions de fonctionnement.

M. Boccherot met en lumière linfuence du relard de bi d.sistribution sur les oseilations dues aux roigulateurs, of indique qu à cet égard lo pronlème est lotaloment diffírent, selon quil s'agul de groupes dectriques isolés ou couples.

Séance du jeud 17 décembe. -- Ta parolo est donnex

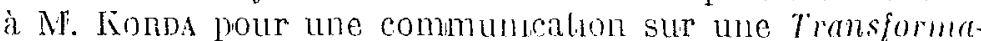
hice Heyland-Korda. Cet apluareil es! un convertisseur "unipolaire "exposé au stand de la Soctéte Assacmenne, dy rui sert à transformer une puissance de 150 kulowalts entrint aiternatuf diphasé à haule tension $(6.000$ volts $)$ à ao puintodes on courant conthu à 120 volls, a 1.500 tours par minulo ('t pôles). M. Konot montre rue ces machunes peureut étre du type asynchrone ou symehrone. Je rotor esl, dans Jes deux cas, conshlué comme celui des moteurs à "rage flér"lrouil ". Il y a aulant de cages " emboildies " l'mon dans l'aulre que de spires "unipolaires" (fue l'on desire mellre rll serie par les conducleurs placrs dans les cncoehes du stator. De charue coté de la machme est placée une bohnu coaxrale pour lexcrtalion à courand conlinu. Leur flux jasso par la section des bagues, contre lesquelles soul placés les frolleurs à courant continu.

M. Gratzmolder présente une communication sur les moteurs de traction à courant alternalif simple. 11 oxamine les sohémas fondamentaux des différents moleurs de hactont a collecleur et, pour cela, il élablit quolques principes merssaires à la bonne commutation of supmose ru à foreco dedromolrice nulle dans la spore en commulation, la commutiton scra suffisante. A cet effed, il faut créer dans la riguon des barres de la spirc en commulation un chaming de whise convenable nour combattre la force dectromotrice statumu? induite par le champ clonl laxe est perpendiculane au phan de la sectuon commulée. (Se champ de conmulabon pouryil itre fourm, soll en emprumlant la puissance magnélisamle nocessaire au réscau, soit en le crénnt par le rolor lu1mème. Ceci condul à neul schémks prnnclpax qui so raminent soit au moteur sério, soil au moteur rémision pripfectionné.

M. Marius Latoun prescule quelques observalions an sujet

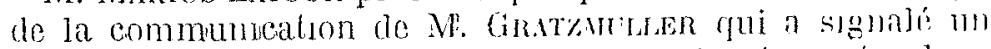
disposutif tès ingóneux pour diminuer la lension chlde dens lames consécutaves du collecteur.

On peut remarquer anil nst pos-ible dohtenir le mine risultat avec un enroulement imbraque double ol en comvant deux lankes, co rut est gethéplement le cas. Finfu, daus los machines mullupolates, on oblicul le mime resullat, srime à un commulateur spocial, ainst que lindirue $\mathrm{V}$. Brumswich: ce procété a été aulrefous appliqué par M. Desoroms. Dalls mo machine à quatre jồes ayant un conroulenont mbriqué

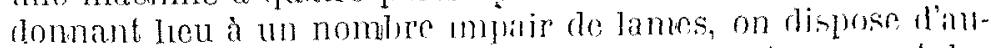
lant de lames morles gue de lames vives, of on folnt les

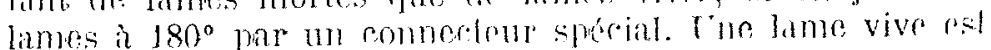

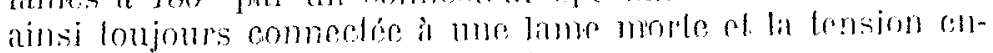
tro lames se trouve ansi diriair. 


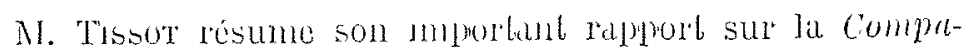
raison des dullérents systèmes de traction.

AK. Dis Tha el Jatuen font queluues observations : le prenier, sur lublité dajouler un lerme on $v$ à la lormule de N. Fanche, ef mome un lerme en $v=$ dans les twmels ; lo deuxièmo, sur la récuprération avec le molour à courant allernalil smuple. (Lo molcur à répulsion conpuensé peut ôtre simplement monté en shunt, ol le moteur serte avec une exctation séplarćej.

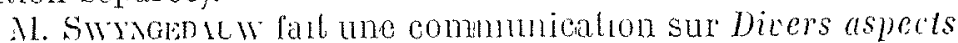
ciconomiques des transports dinergue ob montre que lon peul envisager la question solis trons aspects : de rédunc au minnmun la dépense ; de rendre maximum lo bénéfice, ou de rendre maxmmum lo hux dinlérel. La sechon la pius économique, la plus profilable ou la plus productive eat lia mòme dans le cas où la puissance utile esl dounce ; ces sechons diferont dans le chs ou la panssmee génćratruce est domnce et entèrement ulibsable (cas do chute deau). La fuestion na pas de sens prócis dans le cas dusues à puissalico variajole.

Sćance du vendredi 18 seplembre. - la parole est domnéc à M. Lrgounz qu présenle un résumé de son rapport sur La traction ilectrigue sur les canaur.

Après avorr rappelé que les apparels essayés pour la traction sur les canaux sont principalcment les locomotives sur rals ct les tracteurs monorals of que, scules, les locomolives ont donné lreu à dimportanles apphicalions pratrcues, il aplelle latlontion sur la défimition de la purssance des moteurs, sur les condilions spéciales du démarzage el sur lemplor du courant alternatif smple.

N. Boricinnot fall ensuite une communtcation sur une Nouvelle classe de machines électriques à créer. Le tilre de colle communucation est vrament irop modeste pusquion pouval déja vor en fonclion, dans le Palais de l’énergie, deux de ces nouvelles el inléressantes machmes : le Caposcilla et le Molosenlla dont nous parlerons ultérieurement. Ces nuachmos reposent sur I ulilisation, par des moyens lres simmles, des mouvements oscillants qui sont obtenus loub nalurellement à l'arle de courants alternatifs.

NI. De Grikas domne leclure de son rapport qui résume les siplugues que lon preub fiure concernanl lo fonchonnement des moteurs de trachon de chemins de fer el de lignes métropolntaines dont les condilions de trafic sont lelles, que cos moteurs sont en continuolle surcharge.

Deux progrès réalsés ces teups dernuers ont déjà amélioró le fonchonnement des moteurs, savour : Leur ventula-

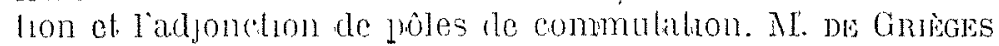
monlye quil reste cneore des.perfectionnements d̀ apporter jour dummer lo coul de lentretien des moteurs, qui est encore beaucoup trop elevé.

M. DE GRuegss émet un voru relalif à la détermination de la puissance des moleurs de traction gui, à son avis, est mstuffsante; sl lon arrival à fixer la clurée des essius à huil ou dix heures, on reminderat à la lonflance ruont les explotants de surcharger les moteurs of on arriverab anst à abaisser d'une facon très intéressante les rans dientrelien.

Sur la proposition de M. DE (imrises, la 5 " section émet le vall sulvanl:

Pont les moteurs de traction. la yo section, estimant que la puissance do ces moteurs est incomplèlement défnie par la scule condition de salusfire uniquement à lessai défni par le Congres de Milan, émel le veru de roir compléter cet essai par un aube dont la durie serte choisie suivant le service rél ruaura ì assurer le moleur dans charne cas.

N. SnI Mali ajoute quelques mols au rapport de M. DE

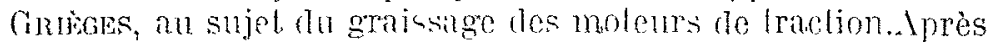
avour cherdié à ulliser lo gmissage à hagues, on a du y renoncer par subte des pertos a'huile, lues à cr que l'échauffonenl des paliors rendaut celle huile excessivement flujde of ruiau houl de tres peu de temps il non reshail ptus dans la boile de graissago. On a arloplé défmilnement le grals. sage par déchels de laine.

M. Sesutrowicz fat remarquer que, protr cléterminer completement la pussance d'un moleur de trachon à courunt contmu, il fautrust theorifuoment posséder :

$L^{\circ}$ Une mesure de la capacilé thermarue du moteur, résul. hunt de lessar dil "d'une heure " acluellement admus (déler. mination du régune dommant $75^{\circ}$ déchauffement pendaul une heure, par lessaı "amćrıcan " ou, plus cxactencnt, du régime " normal "défin a Congrès de Mllan,

$2^{\circ}$ Une mesure de la capaculé démission de chaleur du moleur en service contmu. colle-cr, llomnée avoc assez dexachude par l'essar de dix heures pormeltant de détermmer h pussance " contunue ", loile quelle a clé défune à Milnum.

$3^{\circ}$ Cne mosure de la surcharge maxma momentancment admissmble pentant un lemps lrès courb (correspondant it la " pussance instantauke " dófine à Mhlan). Lintensulé maxma est hmice par la commulalion dans les moteurs orknares. vrec les molours à póle de commulituon, dautres

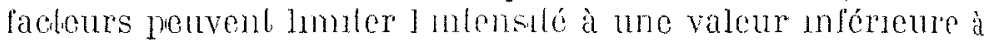
colle mposéc jar la commulation : par exenple l'échauffment do certanes parlıes des enroulemenls, la résistance au pounl de vue mécanifue de cerlanes parlıes du moleur. $4^{\circ}$ Enfin los peries dínergie du moteur aux différents régimes de lension aux bornes et a intensilé (perles ohmiques - pertes au collecteur - pertes dans le noyau dindul - pertes inlermes par frollements!

on peul, avec cel ensemble de renseıgnenents, détermmer exactement l'éclauffemenl d un moleur en serveo, quel]s que soil son ulilsation. Au pout. de vue commercial les tron essals dófinis au Congrès de MHlan el rappelés ci-clessus caractérisent suffisamment un moleur et il semble que lon devralt se borner jour le moment à les adopter.

Flant donnée limportance des arrêts sur la consommalion signalée par M. de Griòges, M. nF J'R 17 falt observer. que la récupération au freinage serait aussı intéressante pour les métropolitains que la récupéralıon en pente jour les chemuns de fer de montagne. Il siguale les efforts lentés dans ce selus.

M. De Traz résumo son rapport sur l'emploi de l'énergie blectrique dans los uines, atehers et munufactures, envisan geant successivement les avantages, los conditions et les divers modes de cel emploi. Il termme en proposant le voeu suivant qui est adopté :

La $5^{\mathrm{e}}$ section émet le voru que les constructeurs de machines-outils, métiers et mecanismes divers employés dans les ateliers, contunuent à dirigcr leurs efforts vers la création dun malérlel nouveau permeltant de twer, des avantages de la commande électrique, meilleur parti quiave le matérel créé et mis au point pour étre commandé par arbres de transmission auquel on se conlente trop souvent d'accoler le moteur électrique.

M. DE Gribges dit qu'il y a en effel mlérét a ce que les fabricanls de machnnes-oubls s'occupent do la question rlo l'aconuplement des moteurs avec leurs machnes. Il signale les difficulth roue cerlames grandes Compagnies ont rencontré de la parl des plus imporlanls fournisseurs de machines-outils pour loger les moteurs dans les bâtis des machines, disposituf qui présente de nombreux avantages de sécurité pour les ouvriers d des garanlies de bon fonctionnoment.

M. Sogum dat qu'il a appliqué, repuis quelques lomps, la chaine pour la commande ólectrique des pompes agricolos.

\section{SIXIENIE, SFCTION

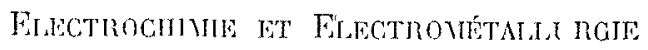

Président : M. GaLt.

Fre-présidents : IMN. CHALרir, BLoxdix.

Secrétaire: M. Jouve.

Síance du mardi to septembre. - Mr. Vutwiven donno locture de son b́lude sur L'blectromélallurgio du cuime. Tes 
conclusions tirées des expériences industrielles de MM. Kellor. Gm, Vittreı et Tavernier, sont que l'électrométallurgie du curve est économique. L'électrolyse du cuivre n'est pralique que si les cuivres employés titrent déjà 98 pour 100 , fandis que le traitement des mattes est peu pratique, ainsi que celul des minerais directs, le tratement des liqueurs dul lovagge des minerals présente certains avantages.

M. Gall cile l'inférêt des Iravaux de M. Keller, augmenté par ce falt que certams minerais pauvres titrant 4 pour 100 , no youront ôtre traités que par les procédés électrométallurgugues.

M. Buovwin a la parole pour son étude sur La fixation de lazote atmosphérique. Après une présentation historique de la question, M. Blondr expiose les procédés de MM. Kowalsky et Moscicki, puls ceux de MM. Birkeland el Eyde ; "eux-ci sont caractérisés par l'utılisation d'arcs alternatıfs lo près de $2 \mathrm{~m}$. de diamètre en forme de disques. Liusine do Nottoden (Norvège) ulilise ces demiers procéidés par l'apheation de $36000 \mathrm{kw}$. à cette fabrication, avec un rendement de $500 \mathrm{~kg}$. d'acide nitrique par kilowatl-an.

Ia fréquence est de 50 périodes par seconde, mais la fréruence n'a jas d'importance, d'après M. Birkeland, qui n'a bas obtenu d'amélioration par l'augmentation de la pérıodalé Il semblerait que l'action ionisante n'a que très peu (1) mlluence, environ 1 pour 1000 .

M. Broxbin expose également les procédés Frank et Caro le fibrication de la cyanamide de calcium fabriquée à Volre-Dame-de-Briançon. Il ature l'attention sur la fixation iu moyen de leffluve.

M. Devaux attire l'attention du Congrès sur les procédés ( ion] ow of Mitckievitch. Ces professeurs emploient à SaintPétersbourg un courant triphasé dans des fours de $14 \mathrm{kw}$. sous une haute tension. Le maximum de rendement corresfond aux proportions de 2 ou 3 pour 100 d'oxyde azotique (n) volume, le rendement augmente avec la vitesse du coumol gazeux, mais seulement jusqu'au chiffre précédent. Le rendement obtenu est de $180 \mathrm{~kg}$ par $\mathrm{kw}$-an. Ce procédé est cncore à l'ébude pratique.

M. Flusin attire l'attention sur les procédés Pawling, (linsbruck, en exploitalion prochaine en France, basé sur le système du parafoudre à cornes, avec des fours de 500 à $700 \mathrm{kw}$, caractérisé par la production directe d'acide azotique $36-40^{\circ} \mathrm{B}$.

MM. Courtors et Dony-Henalut prennent part à la discussion, et ce dernier signale tout particulièrement les recherches de M. Haber, die Carlsruhe, expériencies effectuées dans lo mème ordre d'idées au laboratoire de M. Don'y, à Bruxelles. Le principe d'augmentation du rendement est d'élever laction ionisante de l'arc et, pour cela, il est indispensable dabaisser aussi la température, la température élevée étant un des grands obstacles à cette influence. Le professeur llaber a réalisé ce flait en diminuant légèrement la pression, et en utilisant des électrodes en produits NerNst. Liaction ionisanle est indubitable, car le calcul thermique théorique indiquerait $4000^{\circ}$. La cioncentration en oxyde qui est de 5 pour 100 avec le procédé Birkeland, monte à 14 pour 100 avec les études Haber.

M. GaLl demandant si les procédés Haber sont entrés dans le domaine industriel, M. Dony répond qu'ils sont trop ricents, et que cette question est encore à l'étude.

M. Courtors spécifie que dans les procédés Birkeland (cost la température élevée qui empêche l'ionisation.

M. Gall demande à M. Dony, en dehors de la teneur, de combien le rendement par kw augmente. M. Dony ne peut répiondre exactement à cette question et ajoute quelques renseignements intéressants sur l'arc Birkeland.

Séance du mercredi 16 septembre. - Cette séance a consisté en une visité aux stands des fabricants de produits ćlectrochimiques, à l'Exposition de Marseille.

Séance du jeudi 17 septembre. - La première com- munication est celle de M. GaLs sur La cyanamide calcique, après l'exposé de l'historique de la fixation de lazote par le carbure de calcium. Le procédé Frank et Caro cst appliqué industriellement à Notre-Dame-de-Briançon. M. Gall. explique de façon détaillée les procédés de fabrication, el termme en montrant que le procédé cyanamide a un meilleur rendement au point de vue fixation d'azote, puisque lo rendement correspond ì $2000 \mathrm{~kg}$ acide $n$ trique, landis que les procédés de fixatıon directe par larc atteignent seulement $500 \mathrm{~kg}$ par kilowall-an.

M. Chaumat expose le résultal de ses recherches personnelles sur La réduction électrolytique de lindigo, qui permettent d'obtenur la réduction de l'indigo avec un rendement presque théorique. De plus, les avantages économiques sont énormes, eu égard au prix de revient, comparativement avec les procédés de réduction chimaque ou biologiques. La caractéristıque de ce procédé est l'emploi d'une cathode à grande surface préparée au moyen du graphite enrobé d'indigo bleu.

M. Dony-Henault demandant par quel phénomène l'nndıgo bleu pouvait sortur de la cathode, $M$. Chalumat répond que ses travaux, sans sinquiéter du mode de séparation du liquide de teinture incolore, ont porté sur des dosages dans les liqueurs entourant la cathode.

M. Lacrorx indique qu'ayant étudié lo procédé de M. Chaumat, il a remarqué la facilité avec laquelle on peut remé. dicr à cette question, au moyen de cathodes mases cn circuit au fur et à mesure de la nécessité, et en particulier de l'épuisement du milieu non teignant (nndigo bleu de la cathode).

M. Gr, absent, ne peut présenter son rupport sur L'électrométallurgie et ses progrès actuels ; mais celui-ci ayant ćté imprimé et distribué, il est procédé à sa discussion immédiate.

M. FuUsin reproche à ce rapport d'être incomplet, et de se borner à un exposé historique quelconque. Il cite, entre autres, l'absence de l'étude des procédés Keller, qui travaillent actuellement avec $11000 \mathrm{kw}$, et dont l'importance est bien connue de tous. Plusieurs congressistes s'associent au reproche de M. Flusin.

M. Keller remanque qu'au seul endroit où ses travaux sont signalés, on classe son four comme four à zinc, et s'élève également contre l'absence totale de descriptions d'autres méthodes, dont les siennes. M. Keller s'excuse d'être obligé de prendre la parole sur un point aussi personnel.

M. Keller demaride l'insertion au procès-verbal d'une ébude sur les aciéries d'Unieux. Il exprime le désir qu'en présence d'un rapport de caractère aussi officiel, il se voil obligé de déplorer que les efforts de la Compagnie KellerLeleux et les résultats pratiques obtenus n'aient pas trouvé dans le rappiort quelques lignes de description. Le four électrique d'Unieux, qui a coulé devant le Congrès de l'Association des Mines un lingot de $8000 \mathrm{~kg}$., par application du procédé ci-dessus, est suffisamment caractérisé pour que M. Keller s'autorise à le signaler à l'attention de l'assemblée, puisque M. Gin ne l'a pas fait.

M. F. Clenc rappelle qu'au point de vuc historique, on insiste trop sur le caractère du four de M. Louis Clerc (homonyme de M. Francois Clerc) et des réchauds de la lampe Soleil. I ne faut pas oublier quelle a été l'importance des travaux du regretté maître H. Moissan.

M. GaLL présente son rapport sur Les progrès de l'électrochimie.

M. Dony-Henaulix a présenté un rapport sur les progrès de l'électro-chimie théorique, et appelle l'attention sur les avantages des dosages électrolyliques ef leur rapidité.

Séance du vendredi 18 septemlore. - La première communication est celle de M. AD. Jorve sur l'Etat actuel de l'électrométallurqie et quelques applications nouvelles des siliciures métalliques. Cette communication a été préparée 
par M. Jouve pour compléter celle de M. Gis, reconnue incomplète la vellle. Au cours de celte communication, M. Jouve parle des procédés Côte et Piennon, pour l'électrométallurgie du zinc ( ${ }^{\star}$ ).

M. Chaumat prend la parole sur lozone et son industrue, at présente des idées très personnelles et origmales sur la préparation rabonnelle de lozone.

Aprsè la séance, M. Devaux convoque les congressistes à son laboratorre, pour une expérience intéressante d'application de l'arc électrique.

\section{SEPTIEME SECTION \\ Th́lÉgraphie eT 'TÉLÉPHONIE}

Président : M. Devaux-Charbonnel.

Vace-Irésudent : M. Roosen.

Secrétaire : M. MILON.

Dans cette section, la seule question qui puisse intéresser vrament nos lecteurs est celle que $M$. NEu a traité le mercred 16 septembre, sur La transmission des signaux sur les lignes industrielles au moyen de courants à fréquence clevée $\left.{ }^{\star \star}\right)$.

\section{HUITIEME SECTION}

ENSEIGNEMENT ET Mesures

Président : M. P. JANET.

Vice-Président : M. Barbillon.

Secrétaire : $\mathrm{M}_{\mathrm{L}}$ Jouvion.

Séance du mardi 15 septembre. - M. JANET donne leclure du rapport de M. Durand, sur Les appareils de mesure uctuels, puis l'ordre du jour appelle la communication de M. Campos, sur Les transformateurs de mesure. Les deux types de transformaleurs qui font l'objet de la communcalion ont été étudiés par l'auteur en collaboration avec M. Nobilı, et construils par la maison Olivetti, de Milan.

Se basant sur l'égalıté presque absolue des ampères-tours primaires et secondarres dans les transformateurs usuels, Ggalité qui crée dans les noyaux un champ magnétique faible, l'auteur emploie des circuits magnétiques massifs ou jeu divisés. L'augmentation des pertes dans le fer a une influence favorable sur la différence de phase des courants primaires et secondaires. La présence d'un entrefer convenalılement aménagé permet d'assurer la constance du rapport de transformation.

Le premier type de transformateur construit d'après ce principe utilise, comme circuit magnétique, la boîte en conte qui le renferme et son couvercle. Les enroulements sont disprosés sur un noyau massif venu de fonte sous le couviercile et séparé du fond de la boîte par un entrefer. Le second type, destiné à la mesure des haules intensités, a soll noyau formé d'un câble fiexıble en fil de fer dont les extrémités sont réunies par un joint démontable. Lienroulement secondaire est disposé sur ce câble et protégé par un écran magnétique en cuivre convenablement seclionné.

Le transformateur embrasse le cable parcouru par l'intensité de courant à mesurer. La répartition du courant primaire à l'intérieur du transformateur n'a pas d'influence sur les mesures. Ce transformateur a été réalisé pour mesurer un courant de 25000 ampères, et étudié pour un courant de 40000 amperres.

Séance du mercedi 16 septembre. - Le début de celte séance est consacré à la Discussion du rapport de $\mathrm{M}$. DuRAND sur Les apparenls de mesure actuels.

M. Heinaisch estime que le rapport de $M$. Durand devrait marquer le commencement d'une entente entre le fabricant et l'ingénieur-conseil. Il voudrait que les résultats des essais

(*) Ces procédés ont été décrits dans La Houille Blanche de décembre 1908.

(*) Voir La Houille Blanche de juin 1900. faits dans les divers laboratoires solent publiés, et servent à guider les acheteurs. Il préisente ensuile quelques observalions de détails sur les points suivants : reissorts et syste. nes de mise au zéro; shunt ct leur chute de potentiel; cordons souples. Influence des champs magnétiques extérieurs.

M. FABRY donne ensuite lecture de son rapport sur Les compteurs électriques, puis la discussion est ouverte aus. sitòt.

M. Laporte siassocie à la distinction proprosée par M. FABry des compteurs d’abonnés, el les compteurs de stations centrales. Il signale ensuite l'influence magnétique réciproque de deux compteurs l'un sur l'autre.

Au sujet de l'étalonnement des compteur's sur le couranl normal d'emploi, M. Laporte signale des essais faits par le Laboratoire central pendant vingt-quatre heures avec lectures toutes les cinq secondes. Malgré les difficultés de lectures dans un régime très variable, on peut, avec des expérimentateurs exercés, arriver cependant à une approximation suffisante. MM. BARBiLlon, Garnier et ReNée, signale des mesures de consommation de tramivays concordant à 2 pour 100 près.

M. Mouchard fail remarcuer que le problème est à rísoudre pour les coripteurs à courant aiternatif munis de transformateurs. La discussion s'engage sur cette question et $M$. le présıdent signale des cas où la constante changeail notablement en régime stable et en régime varrable. M. CosSART dit que dans l'équalıon différentielle du mouvement du mobile d'un compteur, la constance des coefficients n'est pas assurée. Un échange de vues a lieu ensuite entre M. Gossar't et M. Fabry sur la méthode calorimétrique signalée par M. Fabry pour l'étalonnement des compleurs.

Au sujet des compteurs d'abonnés, M. Rríć signale les vérifications fartes à Paris au secteur de la Compagnie Parsienne d'Flectricité. On a constaté environ 0,6 pour 100 d'arrèts accidentels. M. BARBILLon signale les variations de constantes après la pose, et insiste sur les gains réalisés dans l'exploitation par une bonne vérification.

M. Dubors présente quelques observations sur la compensation du coefficient de température par un shunt magnétique en acier au nickel. Cie procédé, mis au point par M. Meylan, donne un coefficient de température très varıable $\left(0,5\right.$ pour 100 pour une variation de $\left.30^{\circ} \mathrm{C}\right)$.

Sur les apparells thermiques, M. Dubors signale un procédé de M. Meylan permettant de réduire la consommation des voltmètres à 0.1 ampère, en chauffant indirectement le fil dilatable par un fil fin enroulé tout autour et parcouru par le courant. M. Heinsisch présente quelques observations sur cette communication, à propos du coefficient de température dî̀ aux ressorts.

Séance du jeudi 17 septembre. - M. BRoca fait une Communication sur Les courants de haute fréquence. Après avoir rappelé les mélhodes courantes de mesure des courants à haute fréquence usuels ot la précision obtenue (2 pour 100 environ), l'auteur parle des difficultés qu'il a renciontrées dans la mesure de courants d'une intensité de 20 a. environ. La nécessité de posséder un shunt sans selfinduction l'a conduit à le constituer par des fils symétriquement tendus sur deux joues en ébonite. Liétude de la dilatation cl'un de ces fils renseigne sur la valeur du courant passant dans l'ensemble. L'auteur a vérifié aveci du courant continu et du courant de haute fréquence la constance du pouvoir multiplicaleur du shunt ainsi constitué.

M. Swyngedauw fait une communication sur une Modification de la méthode de Mordey.

M. NèGre communique une note sur la méthode de MM. Marcel Deprez et Routin pour la Détermination des pertes mécaniques des machines électriques.

M. DELoN décrit un Appareil destiné à obtenir de grandes différences de potientiel continues. Après avioir rappelé la nécessité qu'il y a d'effectuer un certain nombre d'essais à 
haute tension avec une différence de polentrel continue, lauteur falt la théorie de son apparell basé sur la charge instantanée du condensaleur, au moment du passage à ses maxima et mumima d'une différence de potientiel alternative. La différence de potientiel contınue ainsi obtenue est gale à envirou deux fois la valeur maxima de la dufférence de potenticl alternative. Suit la présentation de quelques résultats oblenus.

M. Anmagnat résume son rapport sur Liorganisation d'un laboratoire inaustriel.

M. le Présidient donne lecture du rapport de M. BLONDLL, sur ce que doil êlre lingénieur électricien.

Síance du vendredi 18 septembre. - M. Gossart dit quelques mots sur une souscription ouverte pour commémorer la mémoire d'Andró-Marie Ampère, et propose le vou survant, qu est adopté à l'unanimité.

Ira $8^{\circ}$ section émet le vou que le Congrès veulle bien prendre la résolution suivante :

Le Congrès internalional des électriciens réunis à Marseille, s'associant à l'udée touchante des habutants de Poleymieu. de faire de la maison natale d'A.-M. Ampère, devant latuelle als veulent dresser sa statue, un centre de vénéraflon mondiale, engage ses membres à envoyer leurs souscrlulions à cetle belle cuvre et à susciter des souscripteurs lans leur rayon d'influence ".

NL. IIEInRICH, sur L'élément étalon Weston, donne d'abord quelques détalls sur l'histoire de celte pile, el les recherches quu Jui ont donné naissance ; lidée qui y présida fut d'obtenir une prle ayant une force électromotrice mdépendante dè la température. Dès 1891, cet élément, avec coefficient de lempérature négligeable, était présenté au Congrès de Franciort et, en 1895 , celte pile fut désignée sous le $110 \mathrm{~m}$ l'élément au cadmium, englobant la pile originale à soluuon saturée à toutes températures, et un autre élément en délivant. M. Heinrici présente une série d'éléments montrant les différents types créés et nerfectionnés successivement.

Il donne les valeurs des forces électromotrices des élémonts défnalifs et parle du coefficient de vieillissement de ces piles, cocfficient très petit. Quant au coefficient de température, il est très faible (environ 5 millionièmes par degré c.) et peut même changer de signe survant la saturation. La valour absolue de la force électromotrice est constante à 4/10000 près.

M. le Président fait remarquer que les conclusions de M. Henrich sont confirmées par les travaux du Laboratoire central d'électricité, et cite à lappui l'observation d'un élément non saturé faite pendant une période de trois ans. En ce qui concerne les éléments saturés, il semble, d'après les mèmes travaux, que la variation de la force électromotrice absolue avec le temps soit moins considérable. Il cite a l'appui les valeurs communiquées par le National Physical Laboratory de Londres.

M. Hennich parle ensuite des applications pratiques de la pile normale Weston, et des appareils construits dans ce but. Il présente ces appareils, et insiste sur les conditions pratiques de leur emploi. La précision obtenue est de $1 / 1000$.

M. BARBillon parle de l'emploi de la tere comme conducteur de relour dans les lignes industrielles.

Laulear parle ensuite de l'action inductrice de lignes haute tension sur une ligne voisine à basse tension, et des mesures effectuées par lui sur ce sujet. Les résultats obtenus sur une ligne parciourue par un courant de 30 a. montrent que cetle action est loin d'ètre négligeable.

Sur une observation de M. le président, M. Barbillion déclare que l'effet inducteur de la ligne ne dépend pas de la tension à laquelle elle est soumise, mais seulement de l'intensité qui la parcourt.

La section discute ensuite le rapport de M. BLondeL. M. Blondel a envoyé a $M$. le président deux réponses de MM. Crompton et Parshall au questionnaire qu'il a envoyé et qui constituent la base de la discussion.
M. Swingedauw, en quelques mots, controuve quelquesunes des conclusions de MM. Crompton et Parshall, sur l'universalıté des ingémieurs élechrıcıens, el signale la place prépondérante de l'ingénieur exploitant dans l'mdustrie.

M. BarbiLLIon signale quelyues inconvénients constatés dans le mode d'éducation de l'électro-chımiste.

M. le Professeur R.-H. Snith fait une communication en anglais sur l'hıstorıque de l'éducalion de l'ingénıeur électricien en Angleterre. Liauteur sólève contre la séparation en des branches trop distinctes de son éducation.

M. Heinniar signale qu'en Allemagne la nécessité même de trouver des débouchés à la carrière dingénieur empêche la spécialisation.

M. DE Chatelain signale que les conditions particulıères d'existence de l'mngénıeur en Russie ont amenó une noulvelle école à consacrer par partıes égales le temps d'éducation aux trois branches : - sciences - mécanique - électricité, et que le résultat a justité cette mannère de voir. $\Lambda u$ sujet de l'ingénieur-chmmiste, ll pense qu'll faudralt donner à un chimiste une solide éducation électro-technique.

M. G. Higman G.-A., dil qu'au Canada, les étudiants ont trois années d'études communes, el rue ce système donne des ingénıeurs très compétents.

Mi. Camichel signale que M. Sabatier, dans une école d'électro-chimie, donne à ses élèves, avant tout, une éducation de chimiste ; l'enseignement est dirigé dans ce sens, à la suite d'une entente avec les ingénieurs de li région. Il signale enfin la création d'une section d'ingénieursarchitectes.

M. Gossart cite ce qui se passe à l'Université de Bordeaux - Eicole de Chimie - où l’étude de l'électricité va être introduite pour répondre aux demandes industrielles.

M. Swingedauw estime que la pratique de la mécanique est plus facile à acquérir que la pratique de l'ćlectricité.

Après une courte réponse de $\mathrm{M}$. de Chatelan, M. le professeur R.-H. Smith dit que son expérience lui a montré que les notions élémentaires de mécanique appliquée à la construction des machines rendent plus facile l'acquisition des nowons d'élecitro-technique.

La $8^{\circ}$ Section adopte à l'unanımité le vou suivant, qui lus est transmis par la $9^{\circ}$ Section :

Il serait désirable que les dircctcurs des Ecoles d'Ingínieurs fassent apprendre à leurs élèves les manouvres de. la respiration artifucielle ct de traction rythmée de la langue.

\section{NEUVIEME SECTION}

Applications a limgiène et a la médeciNe

Président : M. BERGONIÉ.

Président d'honneur : M. GARIBL.

Vice-président : M. ANDRÉ BROCA.

Secrétaire : M. le $D^{r} D^{\prime A}$ AncoulT.

Au cours de la séance du mercedi 16 septombre, M. ABnAIIAM présente son rapport sur La sterilisation des eaux et de lair par les procédés électriques qui est duscuté par MM. de Montricher, Broca, Vaudrey.

D'autre part, le jeudi 17 septembre, M. Louis ZACON a donné communication de son rapport sur Les formes diverses des accidents électriques et les moyens de les prévenir. Comme conclusion, M. ZACon présente le voeu suivant, qui est adopté à l'unanimité :

La $g^{\circ}$ Section du Congrès, considérant que l'étude des accidents causés par les courants clectriques est de nature à faciliter la prévention, émet le vou que les pouvoirs publics établissent et publient chaque année la relation et la statistique des accidents survenus en France.

Sur la proposition de M. le professeur Bergonié, la $9^{\circ} \mathrm{Sec}$ tion vote également le vœu suivant :

La $9^{\circ}$ Section émet le vœu que, dans les instructions prévues par le décret du 11 juillet 1907 et par l'arrété technique du 21 mars 1908 concernant les secours à donner aux victimes des accidents par l'élertricité: 
$1^{\circ}$ Il soit prescrit d'avoir recours, autunt que possible, aux manouvres de la respiration artificielle en même temps qu'aux tractions rythmées de la langue;

$2^{\circ}$ Que les manauvres soient prolongées pendant longtemps et du moins jusquà l'arrivée du médecin.

Enfin, le vendredi 18 septembre, M. DE KEating-Hart a donné communication de son rapport sur L'action de l'étincelle électrique sur les tissus.

\section{NOTE SUR LES MODES D'ACTION DE LA CORRECTION DE RÉGLAGE Dans la Régulation des Turbines}

Le phénomène de la correction de réglage est difficilement accessible à l'analyse, si l'on se place dans la généralité des cas. Il est au contraire facile de l'étudier dans toutes ses conséquences, sil'on admet quelques hypothèses simples dont on peut s'approcher pratiquement par une construction appropriée des organes en jeu.

\section{I' ETABLISSEMENT DES FORMULES FONDAMENTALES}

Le plus fréquemment, un régulateur réduit à sa plus simple expression se compose :

Io D'un tachy'mètre, ou pendule, monté sur un axe $\mathrm{AB}$ (fig. I) recevant son mouvement de rotation de l'arbre de la turbine par l'intermédidire d'organes de transmissions non élastiques. Toute modification de la vitesse de la turbine a pour effet un déplacement linéaire du manchon $M$. Si les deux positions extrêmes du manchon correspondent à des vitesses peu différentes ( 10 ) pour 100 d'écart par

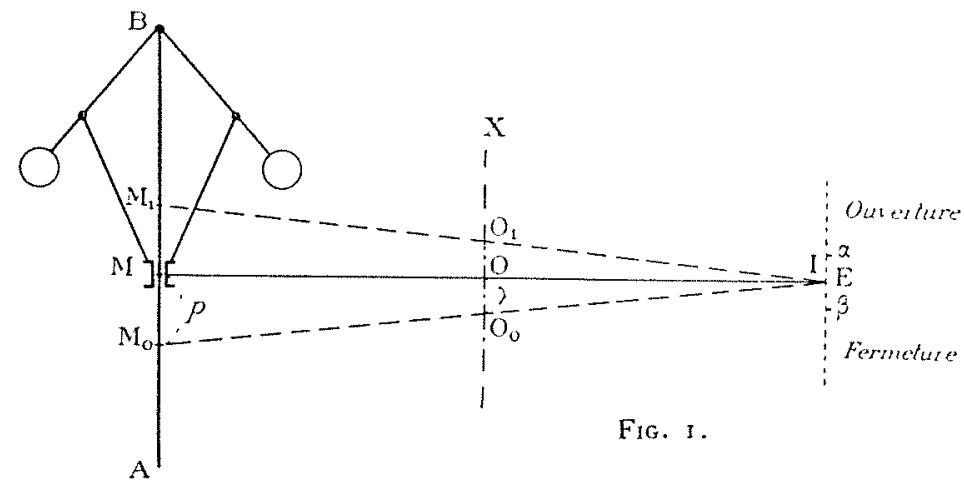

exemple), les déplacements du manchon entre ces deux posi. tions peuvent être considérés comme proportionnels aux pariations de la vitesse angulaire.

Le déplacement $p$ du manchon, compté à partir de la position inférieure, pourra donc se mettre sous la forme:

$$
p=B\left(\omega-\omega_{0}\right)
$$

formule dans laquelle $B$ est un coefficient approprié, $\omega$ est la vitesse angulaire correspondant au déplacement $p$, et $\omega_{0}$ la vitesse angulaire correspondant à la position inférieure du manchon.

$2^{\circ}$ D'un bras de levier MOI, dont une extrémité est astreinte à suivre le déplacement du manchon, et dont l'autre actionne le mouvement de vannage comme il sera dit plus loin.

S'il n'y a pas de correction de réglage, le point d'appui $O$ du levier est fixe. S'il y a correction de réglage, le point $O$ subit un déplacement sur la droite $X$ dans un sens tel qu'il aide l'index $I$ à revenir dans la position de débrayage du servo-moteur actionnant la vanne. Ce déplacement est fonction du déplacement de la vanne, et le plus souvent lui est proportionnel.

$3^{\circ}$ D'un organe, ou série d'organes, formant servo-moteur, tels que tout déplacement de l'index I du levier, en dehors de l'intervalle compris entre deux positions $\%$ et $\beta$ de l'index, occasionne la fermeture ou l'ouverture de la vanne.

Dans le cours de cette étude, l'intervalle $\alpha \beta$ sera supposé réduit à $o$, et $\alpha, \beta$ confondus en $\mathrm{E}$; de plus, la vitesse de fermeture, ou d'ouverture de la vanne, sera supposée constante.

$\mathrm{Si}$, dans ces conditions, l'on prend pour origine des temps l'instant ou commence un régime troublé, la position de la vanne sera fonction du temps d'action du régulateur d'une d'une manière simple. Si $S$ est la section du distributeur au temps $t$ :

$$
S=S_{0}\left[\mathrm{I}-\frac{t_{0} \pm t}{T}\right]
$$

Equation dans laquelle $S_{\mathbf{o}}$ est la section du distributeur à pleine ouverture, $t_{0}$ le temps que le servo-moteur a mis avant le commencement du régime troublé actuel pour amener la vanne de la position de pleine ouverture à celle qu'elle occupe au commencement du régime troublé actuel, c'est-à-dire au temps $t=0$, et $T$ le temps que le servomoteur met pour amener la vanne de sa position de pleine ouverture à la position pour laquelle le travail de la turbine, tournant au nombre de tours normal, est entièrement absorbé par les résistances passives.

Les deux signes \pm de l'équation correspondent : Le signe supérieur à l'action de fermeture, le signe inférieur à l'action d'ouverture.

Equation du régime troublé. - L'équation de D'Alembert, appliquée aux corps tournant autour d'un axe, s'écrit

$\frac{\mathrm{d} \omega}{\mathrm{d} t}=\Sigma \Re_{0}{ }^{t}$ forces motrices $-\Sigma \Re_{\mathrm{ot}}$ forces résislantes.

équation dans laquelle $\omega$ est la vitesse angulaire au temps $t$, et $I$ le moment d'inertie d'un point matériel situé à un mètre de l'axe de la turbine; tournant autour de cet axe avec une vitesse de rotation égale à $\omega$, et de masse telle que sa force vive soit équivalente à celle 'de l'ensemble tournant que mène la turbine. Cette valeur de $I$ est connue pour une installation donnée, et peut être augmentée par l'adjonction d'un volant.

Forces motrices. - Le débit de la turbine sera supposé proportionnel à la section du distributeur (condition réalisée pour les turbines à libre déviation, et non dans les turbines à réaction, dont le forcement varie avec l'ouverture du vannage). Le débit au temps $t$ aura alors pour expression, d'après l'équation (2)

$$
q_{t}=q_{0}\left(\mathrm{I}-\frac{l_{0} \pm t}{T}\right)
$$

$q_{t}$ étant le débit au temps $t$, et $q_{0}$ le débit à pleine ouverture. La puissance de la turbine aura par suite pour expression

$$
P_{t}=\rho_{l} q_{t} H=p_{t} q_{0} H\left[\mathrm{I}-\frac{t_{0} \pm t}{T}\right]
$$

